\title{
Methode für markenübergreifendes Produktdesign am Beispiel eines Akkumoduls
}

\section{Method for cross-brand product design using the example of a battery module}

\author{
Matthias Sebastian Fischer ${ }^{1 *}$, Daniel Holder ${ }^{1}$, Florian Reichelt ${ }^{1}$, Franziska \\ Kern ${ }^{1}$, Thomas Maier ${ }^{1}$ \\ ${ }^{1}$ Institute for Engineering Design and Industrial Design, University of Stuttgart \\ ${ }^{*}$ Korrespondierender Autor: \\ Matthias Sebastian Fischer \\ IKTD Universität Stuttgart \\ Pfaffenwaldring 9 \\ 70569 Stuttgart \\ Telefon: 0711/685-66041 \\ Mail: matthias.fischer@iktd.uni-stuttgart.de
}

\begin{abstract}
Replaceable products for changing use in machines from different manufacturers confront industrial designers with new challenges. In contrast to classic products, a strong product uniqueness must be created here for the recognition of the functional system and an individually modifiable brand allocation so that the product does not look like a foreign body in the application machine. The three-stage design method to solve this problem is placed before the concepts are created. This includes the analysis of the technical degree of freedom of the design, an analysis of the competition and the definition of a design target. In addition to the integration of the method into the product development process, the development of a cross-brand battery module is described as an example.
\end{abstract}

\section{Keywords}

Design Method, Industrial Design, Brand Design, Product Uniqueness, Brand Allocation 


\section{Einleitung und Motivation}

Im Industriedesign ist eine klare Ausrichtung am Corporate Design in Form eines Design Manuals sehr verbreitet, um das Produkt später der Marke eindeutig zuordnen zu können. Doch die Frage ist: wie wird ein Produkt gestaltet, welches in Maschinen ganz unterschiedlicher Marken integriert und sogar zwischen diesen ausgetauscht werden soll?

Austauschbare Akkupacks für Elektrohandwerkzeuge besitzen z. B. bereits eine markenspezifische Modularität und eine einheitliche Designsprache. Eine Erweiterung dieser Modularität stellen standardisierte Akkuwechselkonzepte dar, wie sie zum Beispiel von GreenPack [1] für urbane Mobilitätskonzepte angeboten werden. Solche Akkumodule können von unterschiedlichen Herstellern in ihre Produkte als wechselbarer Energielieferant eingebaut werden. Die Akkus erfüllen als separat betrachtetes Produkt keinen Zweck. Sie benötigen ein Anwendungssystem (bzw. eine Anwendungsmaschine) in dem ihre Funktion benötigt und erforderlich ist. Bei Akkupacks von Elektrohandwerkzeugen sind Markenzuordnung (für die Einordnung in das Produktsystem der Marke) und Produkteigenständigkeit (für eine ästhetische und differenzierbare Gestalt) aufeinander abgestimmt. Als problematisch stellt sich zum aktuellen Zeitpunkt jedoch die visuelle Integration der markenübergreifenden Akkumodule in einer Anwendungsmaschine dar. Für den Nutzer darf der Akku nicht als Fremdkörper erscheinen. Hier muss eine Integration des Produktes in unterschiedliche Markendesigns gewährleistet werden. Es besitzt demnach kein eigenes Markendesign, sondern eine hohe Produkteigenständigkeit in der Gestalt zur Einordnung in das anwendungsübergreifende offene Produktsystem. Ein bereits bestehendes Beispiel ist die Normbrunnenflasche für Mineralwasser und Limonade. Diese lässt sich von den Getränkeherstellern mittels Etiketten individuell anpassen. Das Produktdesign wird von den Nutzern akzeptiert und sogar auf modernere Kunststofflaschen übertragen. Ein weiteres Beispiel sind Aufbewahrungs- und Transportboxen für professionelle Handwerkzeuge. Das Produkt hat durch eine hohe Wiedererkennbarkeit einen eindeutigen Systembezug. Zugleich ist aber auch (mittels geringer Modifizierungen) eine Eingliederung in das Markendesign des Getränke- oder Werkzeugherstellers möglich. Dieses Entwicklungsziel wird selten anvisiert, ist aber für ein markenübergreifendes Akkumodul erforderlich. Mit unserem neuen designmethodischen Ansatz wollen wir uns diesem Spannungsfeld annehmen und den Entwicklungsprozess eines modularen Akkuwechselkonzeptes vorstellen. Diese Arbeit wurde im Rahmen des Forschungsprojekts „BaSyMo“ (Batteriesystem für Modularität) vom Bundesministerium für Wirtschaft und Energie gefördert.

\section{Hintergrundinformationen}

Dieses Kapitel soll einen Überblick über die wichtigsten Grundlagen der Produktgestalt, Industriedesign, Produktentstehungsprozess und markenbezogene Produktgestaltung bieten.

\subsection{Einteilung der Produktgestalt}

Die Produktgestalt wird nach Seeger [2] in vier Teilgestalten separiert. Die Einteilung umfasst Aufbau (Au), Form (Fo), Farbe (Fa) und Grafik (Gr) (siehe Bild 1) und kann nur bedingt losgelöst voneinander betrachtet werden. Die Einflussnahme auf die Wahrnehmung des Produktes ist jedoch durch jede der Teilgestalten möglich [3].

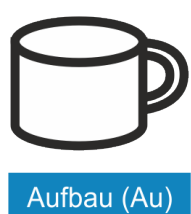

Aufbau (Au)

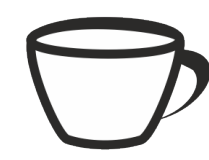

Form (Fo)

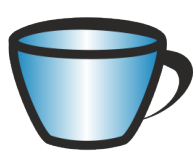

Farbe (Fa)

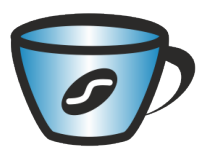

Grafik $(\mathrm{Gr})$

Bild 1: Teilgestalten Aufbau, Form, Farbe und Grafik am Beispiel einer Kaffeetasse nach Holder et al. [4] 
Der Aufbau beschreibt die einfache Zusammensetzung des Produkts aus Grundkörpern wie z. B. Zylindern, Quadern und Prisma. Die Form beschreibt die Flächen, Flächenübergänge und Linien welche die Grundkörper überspannen. Mit der dritten Teilgestalt Farbe wird dem Produkt ein Farbwert inklusive Reflexionsgrad zugeordnet. Die Grafik tritt z. B. in Form von Logos, Symbolen und Buchstaben auf. Die Komposition aller Teilgestalten beschreibt das komplette Produkt und dient des Weiteren einer sukzessiven Gestaltentwicklung im Produktentstehungsprozess (siehe Kapitel 2.2).

\subsection{Industriedesign im Produktentstehungsprozess}

Der Produktentstehungsprozess besteht nach VDI 2221-1 [5] aus der Produktplanung, Produktentwicklung und Realisierung/Produktion. Dabei soll das Industriedesign möglichst früh in den Produktentwicklungsprozess mit eingebunden werden. In der Vorgängerversion VDI 2221 [6] wurde der Produktenwicklungsprozess noch statisch in die vier Phasen „Aufgabe klären“(Planen), „Konzipieren“, „Entwerfen“ und „Ausarbeiten“ unterteilt.

Die inzwischen zurückgezogene Richtlinie für das Industrial Design VDI/VDE 2424-3 [7] orientiert sich an den vier Phasen der VDI 2221 [6]. Dabei ist in der Planungsphase (Aufgabe klären) vor allem der Austausch mit dem Marketing wichtig. Das Marketing stellt dafür beispielsweise das Erscheinungsbilds des Unternehmens (Corporate Design Manual) und seine Stilelemente zur Verfügung. Umgekehrt stellt das Marketing an die Industriedesigner Anforderungen. Dazu gehören unter anderem, dass sich das neue Produkt von Wettbewerbsprodukten unterscheidet, aber sich auch in das Erscheinungsbild des Unternehmens einfügt (mittels einheitlicher Elemente). In der Konzeptphase werden Gestaltkonzepte dann entsprechend der Anforderungen der Planungsphase von Industriedesignern erstellt. In der Entwurfsphase wird das resultierende Konzept geometrisch konkretisiert. Es erfolgt die maßstäbliche Dimensionierung und Ausarbeitung von Details. In der Ausarbeitungsphase wird unter anderem die konsequente Anwendung der Ergebnisse der vorgegangenen Phasen sichergestellt. Die neue Richtlinie VDI-Richtlinie 2424 soll sich an der überarbeiteten VDI 2221 orientieren [8]. Dabei soll auch das markentypische Produktdesign (Corporate Product Design) mehr berücksichtigt werden [8].

Seeger [2] orientiert sich an der VDI 2221 [6]. Er beschreibt, dass in der Konzeptphase der Aufbau festgelegt und in der Entwurfsphase die Teilgestalt Form fixiert wird. In der Ausarbeitungsphase folgen die Oberflächen mit Farbe und Grafik. Auch Wohlgemuth [9] ordnet die Gestaltung von Farbe und Grafik der Ausarbeitungsphase zu.

\subsection{Markenbezogene Produktgestaltung und Markenstrategie}

Marken dienen der Identifikation und Differenzierung von Produkten [10]. Zwischen Marke und Produkt bestehen nach Kreuzbauer und Malter [11] zwei Verbindungen. Ästhetisches Produktdesign kann zu positiver Markenbewertung führen und mit der Markenkategorisierung kann die Überzeugung der Nutzer bezüglich des Produkts beeinflusst werden. Der Markenbezug wird dabei durch ein ähnliches Produktdesign erzielt. Wiederum müssen sich einzelne Produkte für ihre Existenzberechtigung gegenüber anderen Produkten differenzieren [12]. Für die Gestaltung eines erfolgreichen Produktes müssen beide Aspekte ausreichend berücksichtigt werden [13]. Wichtig ist das vor allem bei großen Produktsystemen [14]. In der Literatur finden sich für diese beiden Gestaltungsziele auch die Begriffe Markenidentität und Produktidentität [15].

Es existieren bereits Forschungsarbeiten um Ähnlichkeiten im Aufbau [16] bzw. in der Form, Farbe und Grafik [17] zu bestimmen. Für eine detaillierte Übersicht an Verfahren, mit welchen ähnliche Formen für den Markenbezug ermittelt werden können, wird auf Fischer et al. [18] verwiesen. 
Umgesetzt wird der einheitliche Markenauftritt in der Regel mit Corporat Design Manuals. Allerdings existieren Corporate Design Manuals nur innerhalb einer Marke und können nicht auf markenübergreifende Produktgestaltung angewendet werden. Sie beinhalten vor allem den Einsatz von Farben, Schriften und Logos. Dahingegen werden in der VDI/VDE 2424-2 [19] neben der Farbe und Grafik auch sogenannte Formkonstanten (wiederholbare Formelemente) für eine Markenzuordnung genannt. Wiederkehrende charakteristische Formelemente werden in der VDI/VDE 2424-1 [20] sogar als wirksamer gegenüber Farbe und Beschriftungen beschrieben. Wie mit wiederkehrenden Elementen oder Differenzierungen umgegangen wird, ist unter anderem von der Markenführung abhängig. Nach VDI 4506-4 [21] wird dabei zwischen Einmarkenstrategie, Mehrmarkenstrategie, Dachmarke, Einzelmarke und Familienmarke unterschieden.

\section{Forschungsfrage und Handlungsbedarf}

Im Gegensatz zu den klassischen Produkten mit Ähnlichkeiten auf Markenebene und Differenzierung auf Produktebene (siehe Kapitel 2.3) invertiert sich diese Problematik bei einem markenübergreifenden Produkteinsatz. Eine hohe Ähnlichkeit der Wechselprodukte ist für die Akzeptanz und Vertrautheit von großer Bedeutung. Für die Integration in Anwendungen unterschiedlicher Marken muss allerdings eine Differenzierung im Design realisierbar sein. Aktuelle Betrachtungen der Markenstrategie (siehe Kapitel 2.3) gehen derzeit nicht über die Grenzen eines Unternehmens hinaus. Das Markenmanagement mündet zuletzt immer in einer Dachmarke auf der obersten Unternehmensebene.

Ein markenübergreifendes Batteriemodul entspricht einer unternehmensübergreifenden Mehrmarkenstrategie. Das Design des Produkts ist für verschiedene Marken anpassbar. In der Regel wird nur die technische Basis übernommen und das Produktdesign bewusst differenziert, sodass ein Bezug zwischen technisch identischen Produkten verschwindet. Bei einem markenübergreifend eingesetzten Batteriemodul muss das Produkt jedoch eine eigenständige Wiedererkennbarkeit für den Systembezug besitzen. In Folge dessen steht der Ähnlichkeit der Produkte eine Differenzierung für die Marken entgegen.

Daraus leitet sich die Forschungsfrage ab, inwiefern eine Designmethode für zielgerichtete Markenzuordnung und Produkteigenständigkeit für markenübergreifendes Produktdesign aufgebaut werden kann. Bisher wird die Corporate Design-Umsetzung im Produktentstehungsprozess nur unzureichend betrachtet (siehe Kapitel 2.2), weshalb die Designmethode sinnfällig in den Produktentstehungsprozess eingeordnet werden soll.

\section{Methode zur fokussierten Gestaltung von Markenzuordnung und Produkteigenständigkeit}

Die erstellte Methode gliedert sich in drei Schritte. Im ersten Schritt wird der technische Freiheitsgrad für die Gestaltung ermittelt. Danach folgt die Analyse der auf dem Markt befindlichen Wettbewerberprodukte, inwiefern dieser Freiheitsgrad für Markenzuordnung und Produkteigenständigkeit genutzt wird. Im dritten Schritt findet die Zielsetzung der Gestaltung für die Markenzuordnung und Produkteigenständigkeit statt (siehe Bild 2). Die Grundlage dafür liefert die Einteilung der Produktgestalt in die vier Teilgestalten Aufbau, Form, Farbe und Grafik (siehe Kapitel 2.1).
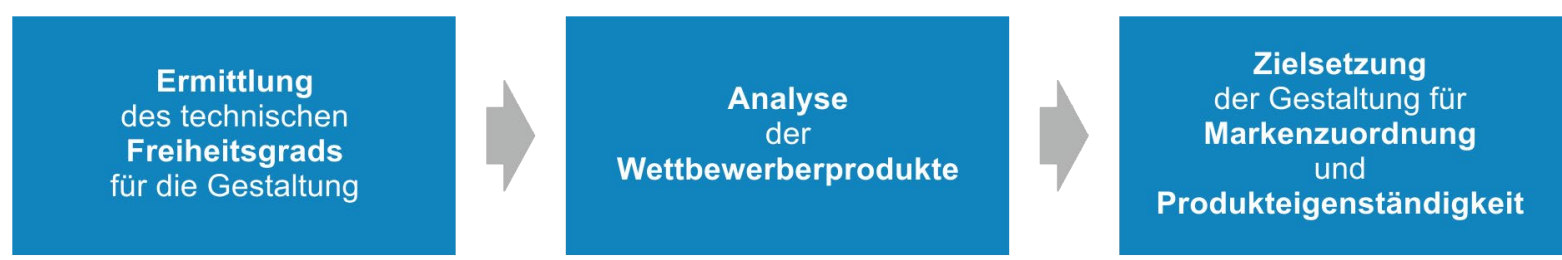

Bild 2: Prozessschritte der Designmethode 


\subsection{Beschreibung der Prozessschritte}

Der technische Freiheitsgrad für die Gestaltung wird in der Literatur als „technischer Freiheitsgrad“ [22], „konstruktiver Freiheitsgrad“ [2], „formaler Freiheitsgrad“ [20] [23] oder "gestalterischer Freiheitsgrad“ [24] bezeichnet. Dieser Freiheitsgrad legt fest, inwiefern das Erscheinungsbild verändert werden kann [19]. Bestimmt wird der Freiheitsgrad vor allem durch die Funktion des Produkts, Herstellungsverfahren, gebrauchstechnischen Eigenschaften (Ergonomie), Standards, Leitlinien, Gesetze und Vorschriften [22]. Dazu kommt noch der Einfluss von technologischen (neue Produkterfindungen oder neue Verfahren) und ökologischen (Recycling) Umfeldbedingungen [22]. Letztlich spielen auch wirtschaftliche Faktoren eine Rolle (Projektkosten und Fertigungskosten für das Design) [22]. Demnach ist der technische Freiheitsgrad für die Gestaltung von dem individuellen Projekt einer spezifischen Firma mit den zugehörigen Randbedingungen abhängig. Neben den Rahmenbedingungen für die eigene Entwicklung und Produktion ist auch der technischfunktionale Einsatz des Produkts in unterschiedlichen Anwendungen zu betrachten. Daraus ergeben sich vor allem Beschränkungen im Aufbau bzw. Anordnung von Funktionselementen. Restriktionen für die Formgestaltung können zum Beispiel durch „Hardpoints“ auftreten [25]. Dabei werden fixe Punkte (auch Grenzpunkte) für die Form definiert. Seeger [2] unterscheidet mit der Analyse des Freiheitgrads die Teilgestalten in variabel und invariable Teilgestalten. Auch Schmid \& Maier [24] adaptieren dieses Vorgehen für das Interfacedesign. Für eine differenziertere Bestimmung des technischen Freiheitsgrads für die Gestaltung wird hier eine Skala eingeführt (0: sehr geringer Freiheitsgrad; 6: sehr hoher Freiheitsgrad). Bewertet wird der Freiheitsgrad vor allem durch die Bereiche Forschung \& Entwicklung, Fertigung und Industriedesign unter Berücksichtigung der unternehmens- und projektspezifischen Rahmenbedingungen.

Anschließend folgt eine Wettbewerberanalyse über die Nutzung des Freiheitsgrads für die Markenzuordnung und Produkteigenständigkeit differenziert in die einzelnen Teilgestalten. In Abhängigkeit des Produkts und der Kunden sind ggfs. unterschiedliche Produktkonfigurationen möglich. Dieser Konfigurationsspielraum kann in der Regel nicht für die Markenzuordnung und Produkteigenständigkeit genutzt werden. Bei der Wettbewerberanalyse der Gestaltung kommt daher noch der Faktor der Individualisierung hinzu. Während bei Konsumgütern häufig nur wenig bis nichts konfiguriert werden kann, ist dies bei Investitionsgütern vermehrt der Fall. Überschneidungen der drei Faktoren bei den Teilgestalten sind möglich. Bei der markenübergreifenden Produktgestaltung muss auch der Anwendungsrahmen, d. h. die für die Integration vorhandenen Anwendungsmaschinen des Produkts analysiert werden. Die Bewertung erfolgt durch Vertrieb, Marketing und Industriedesign.

Im dritten Teil der Methode wird die Zielsetzung für den Einsatz der Teilgestalten festgelegt. Anhand der bestehenden Marktsituation und der Markenstrategie kann gezielt der Entwicklungsfokus in den Teilgestalten gesetzt werden. Idealerweise wird der Freiheitsgrad einer Teilgestalt bisher kaum genutzt und kann somit als Unterscheidungsmerkmal in dieser Produktgruppe genutzt werden. Die Zielsetzung in den Teilgestalten ergibt sich aus der Markenstrategie und es ist prinzipiell offen, ob dem Wettbewerbsfeld gefolgt oder entgegenwirkt werden soll. Durchgeführt wird die Bewertung in der Regel von Vertrieb und Marketing.

Beispielbewertungen von Konsum- und Investitionsgütern bezüglich des Freiheitsgrads, Markenzuordnung, Produkteigenständigkeit und Individualisierung befinden sich in Bild 3. Wie bereits erwähnt sind die Bestimmungen des Freiheitsgrads von vielen unternehmensspezifischen Faktoren abhängig. Die Bewertung durch die Autoren und Experten des Technischen Designs sind somit nicht zwangsläufig auf eine spezifische Produktentwicklung übertragbar und beruht auf zahlreichen durchgeführten Projekten und Literaturwissen. Die Ergebnisse sind mit Netzdiagrammen dargestellt und decken sich mit der Einschätzung von 
Klöcker [23], nach dem der Freiheitsgrad bei Konsumgütern höher ist als bei Investitionsgütern.

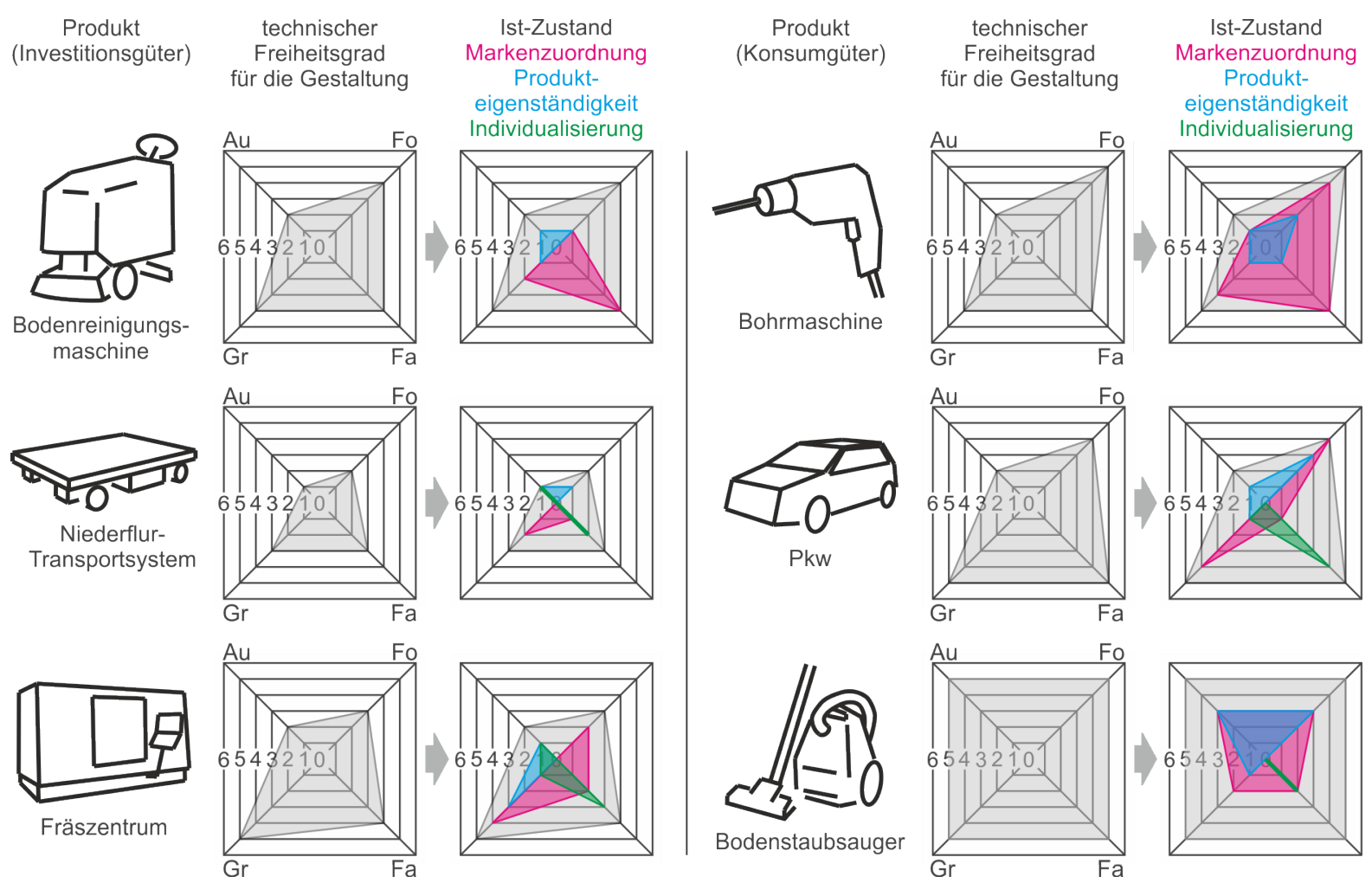

Bild 3: Bsp. Markenzuordnung, Produkteigenständigkeit und Individualisierung in den Exterieur-Teilgestalten (0: sehr geringer Freiheitsgrad; 6: sehr hoher Freiheitsgrad)

\subsection{Einordnung der Methode in den Produktentstehungsprozess}

Die Bestimmung des Freiheitsgrads wird von Schmid \& Maier [24] in der Planungsphase des Produktentwicklungsprozesses verortet. Wettbewerberanalysen werden nach VDI 2221 [6] vor allem der Planungsphase und Ausarbeitungsphase zugeordnet. Nach der VDI 4520-1 [26] werden diese Schritte (inkl. der Zielsetzung der Entwicklung) dem Produktentwicklungsprozess vorgelagert und befinden sich in der Produktplanungsphase (nach VDI 2221-1 [5]). Die beschriebenen Schritte kommen somit in der Produktplanungsphase und der Planungsphase der Produktentwicklung zur Anwendung.

Die Umsetzung der Zielsetzungen erfolgt dann in den weiteren Schritten des Produktentwicklungsprozesses. Dabei kann nicht mehr an der zeitlichen Einteilung der Entwicklung der Teilgestalten nach Seeger [2] (siehe Kapitel 2.2) festgehalten werden. Analog zur VDI 2221-2 [27] muss im Folgenden eine spezifische Entwicklungsreihenfolge generiert werden. Ausgewählte Teilgestalten für Markenzuordnung und Produkteigenständigkeit müssen schon zu Beginn der Konzepterstellung berücksichtigt werden. Soll z. B. die Markenzuordnung durch die Grafik gestaltet werden, kann diese nicht erst in der Ausarbeitungsphase stattfinden. Zudem kann der kreative Gestaltungsfokus in der Konzeptentwicklung weitgehend auf die Teilgestalt für die Produkteigenständigkeit gelenkt werden. Die Einordnung in den technischen Produktlebenszyklus nach VDI 2221-1 [5] ist in Bild 4 dargestellt.

Die Zielsetzungen der Teilgestalten müssen bei den Bewertungen von Konzeptvarianten berücksichtigt werden. Zudem können Korrekturen am Freiheitsgrad durch z. B. Änderung des Herstellungsverfahrens während der Produktentwicklung erforderlich werden. 


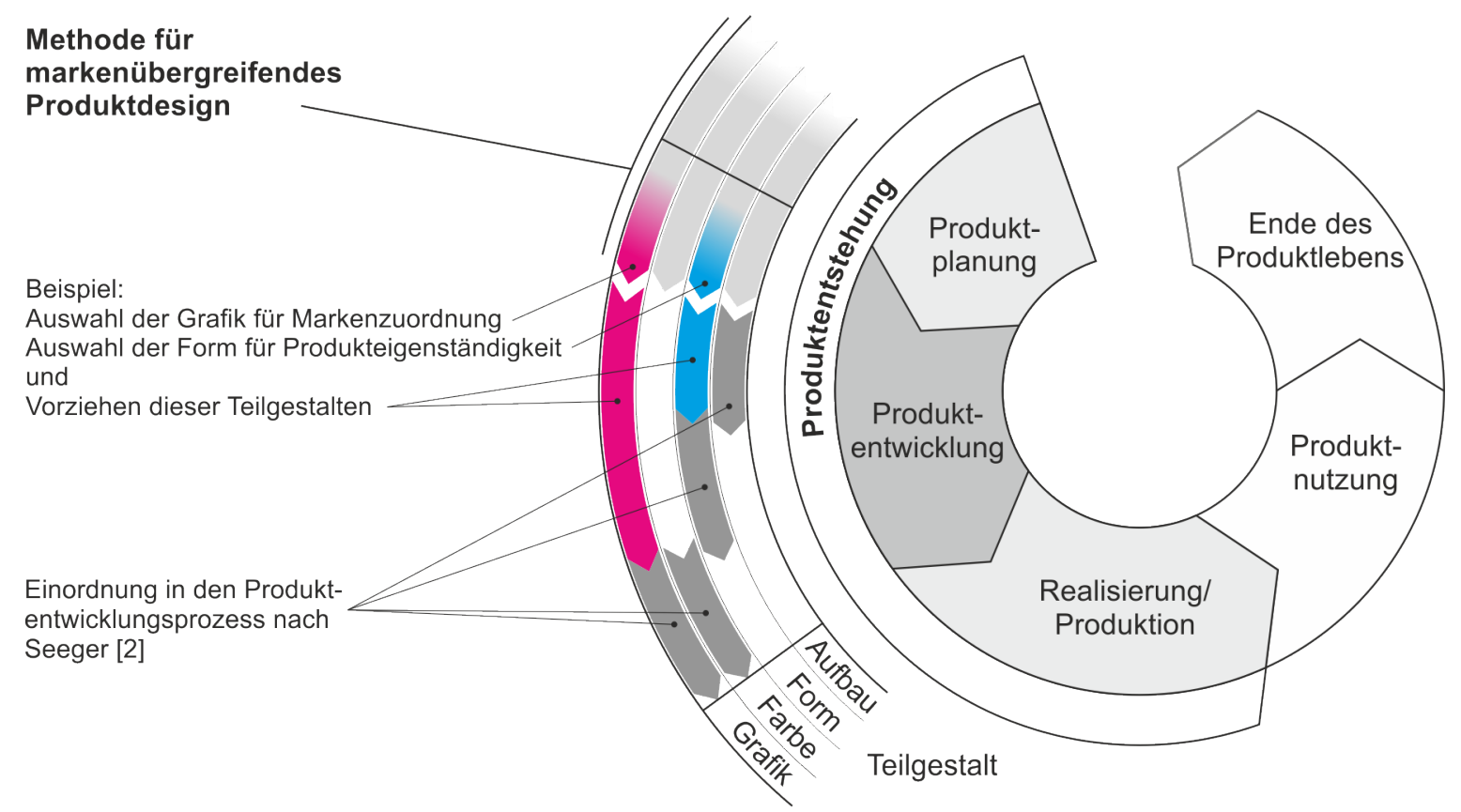

Bild 4: Einordnung der Methode für markenübergreifendes Produktdesign in den technischen Produktlebenszyklus nach VDI 2221-1 [5]

\section{Praxisbeispiel: Markenübergreifendes Akkumodul}

Der technische Freiheitsgrad für die Gestaltung resultiert aus den Anforderungen des BaSyMo-Projekts. Ziel ist ein Akku mit ca. $1 \mathrm{kWh}$ Energieinhalt, was zu einem Akkugewicht von ca. $10 \mathrm{~kg}$ führt. Die im Forschungsprojekt korrespondierenden Akku-Anwendungen sind Reinigungsfahrzeuge und Niederflur-Transportsysteme. Zum Ausblick gehören unter anderem Flurförderzeuge und bei weiterer Verbreitung auch Konsumgütergeräte für den Haushalt/Garten. Die Rahmenbedingungen der hier wichtigsten Kriterien und ihre Auswirkungen auf den Freiheitsgrad sind in Tabelle 1 beschrieben.

Tabelle 1: Rahmenbedingungen des BaSyMo-Projekts und deren Auswirkungen auf den Freiheitsgrad

\begin{tabular}{|c|c|c|}
\hline Kriterium & Rahmenbedingung & Auswirkung auf Freiheitsgrad \\
\hline Funktion & Platzsparender Energieträger & $\downarrow$ Aufbau \\
\hline $\begin{array}{l}\text { Herstellungsverfahren } \\
\text { (für Sichtelemente) }\end{array}$ & $\begin{array}{l}\text { Kunststoffspritzguss (teilweise } \\
\text { Aluminiumdruckguss) }\end{array}$ & $\begin{array}{l}\uparrow \text { Form } \\
\uparrow \text { Farbe }\end{array}$ \\
\hline Ergonomie & $\begin{array}{l}\text { Zwei Griffe an der Oberseite (resultiert aus } \\
\text { Usecase) }\end{array}$ & $\downarrow$ Aufbau \\
\hline Standards & Lithium-Ionen-Zellformate (PHEV2, 21500, 18650) & $\downarrow$ Aufbau \\
\hline $\begin{array}{l}\text { Gesetze und } \\
\text { Vorschriften }\end{array}$ & $\begin{array}{l}\text { Zahlreiche Kennzeichnungsvorschriften für } \\
\text { Lithium-Ionen-Akkus }\end{array}$ & $\downarrow$ Grafik \\
\hline Anwendungsfeld & Quaderförmiger Kasten (300 x 175 × 190 mm) & $\downarrow$ Aufbau \\
\hline
\end{tabular}

$\uparrow$ Freiheitsgrad steigt; $\downarrow$ Freiheitsgrad sinkt

Im Wettbewerberfeld sind nahezu alle Akkus quaderförmig. Eingesetzt werden bei der Teilgestalt Form vor allem plane Flächen. Akkus für Industriegüter sind meist schwarz oder grau (unbunte Farben) und mit Kennzeichnungsaufklebern des Herstellers (Grafik) versehen. Dahingegen sind die Wechselakkus für Konsumgüter in der Regel in einer auffälligen Farbe (z. B. Grün [1]) gestaltet. Bei diesen ist zudem anzumerken, dass eine Einmarkenstrategie mit einem einzigen Produkt zur Anwendung kommt. Dabei sind Markenzuordnung und 
Produkteigenständigkeit nicht mehr zu unterscheiden (Marken- und Produktidentität sind identisch). Individualisierungen existieren bei Akkumodulen in der Regel nicht.

Für die Zielsetzungen der Gestaltung ist neben den Bewertungen des Akkumoduls auch die Bewertung der Anwendungsmaschinen erforderlich (Reinigungsmaschine und NiederflurTransportsysteme). Daher soll für die Produkteigenständigkeit die bisher (bei Akkus und den Anwendungsmaschinen) wenig eingesetzte Teilgestalt Form genutzt werden. Die individuell anpassbare Markenzuordnung soll über farbige Elemente und Bauteile erfolgen, da diese Teilgestalt bei den Anwendungsmaschinen bereits dafür genutzt wird. Die resultierenden Bewertungen für den Freiheitsgrad, die Markenzuordnung, Produkteigenständigkeit sowie die Zielsetzungen sind in Bild 5 dargestellt.

Auf diese Art und Weise wurde eine wenig berücksichtigte Teilgestalt (Form) ermittelt, welche nun für die Produkteigenständigkeit genutzt werden soll. Zudem wird die anpassbare Markenzuordnung in der passenden Teilgestalt Farbe der Anwendungen gewährleistet. Für den Gestaltungsprozess ist die Zielsetzung damit erkenn- und kommunizierbar.
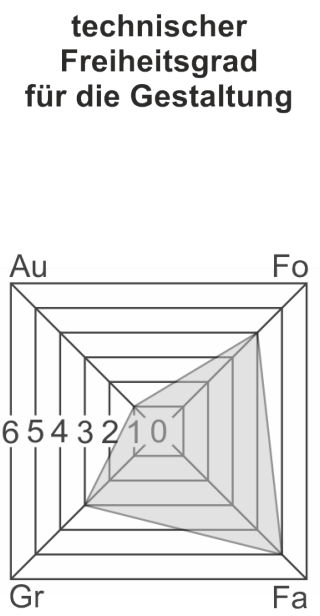

Ist-Zustand

Markenzuordnung Produkteigenständigkeit

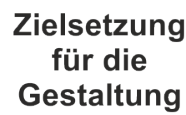

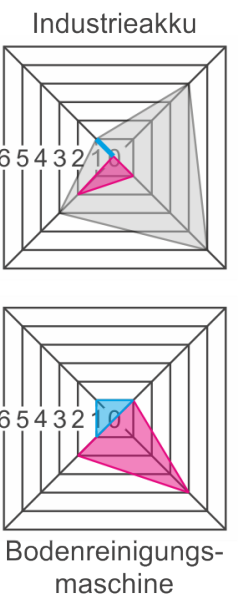
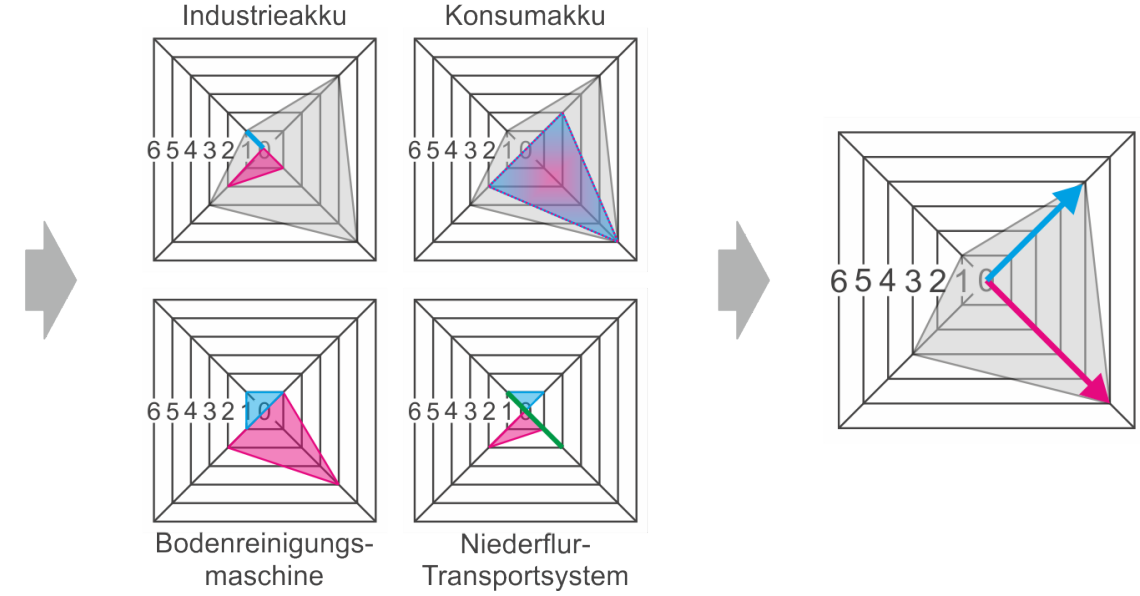

Bild 5: Ergebnisse der drei Schritte der Methode bei dem Besipiel des Akkumoduls (0: sehr geringer Freiheitsgrad; 6: sehr hoher Freiheitsgrad)

Aus der nutzerzentrierten Entwicklung resultiert ein quaderförmiges Akkumodul mit zwei ergonomischen Klappgriffen auf der Oberseite. Dabei werden die Bedienelemente (Klappgriffe) aus Kunststoff für die Markenzuordnung zur markenspezifischen Einfärbung verwendet. Das Akkugehäuse bleibt unbunt, analog zu den schwarzen Gehäusen der Industrieakkus.

In der Konzeptphase wurden neun Formkonzepte bewertet und von den drei ausgewählten Konzepten anschließend elf Formdetaillierungen erstellt. Das finale Konzept wurde durch die Entwurfsphase und Ausarbeitung zur Reife gebracht. Erwähnenswert ist die dabei erfolgte Betonung der Form durch eine nach außen verlegte Verrippung im sogenannten „FlowDesign“. Abbildungen der Konzepte und des ausgearbeiteten Akkumoduls sind in Bild 6 dargestellt. Mittels der fokussierten Konzepterstellung in der Teilgestalt Form wurde schnell und effizient ein markenübergreifendes (individuell spezifizierbares) Produktdesign für den Akku entwickelt. Die Formen des Gehäuses heben sich dabei vom Wettbewerberfeld ab, während die modifizierbaren farbigen Griffe die Markenzuordnung zu den Anwendungsmaschinen herstellen. 


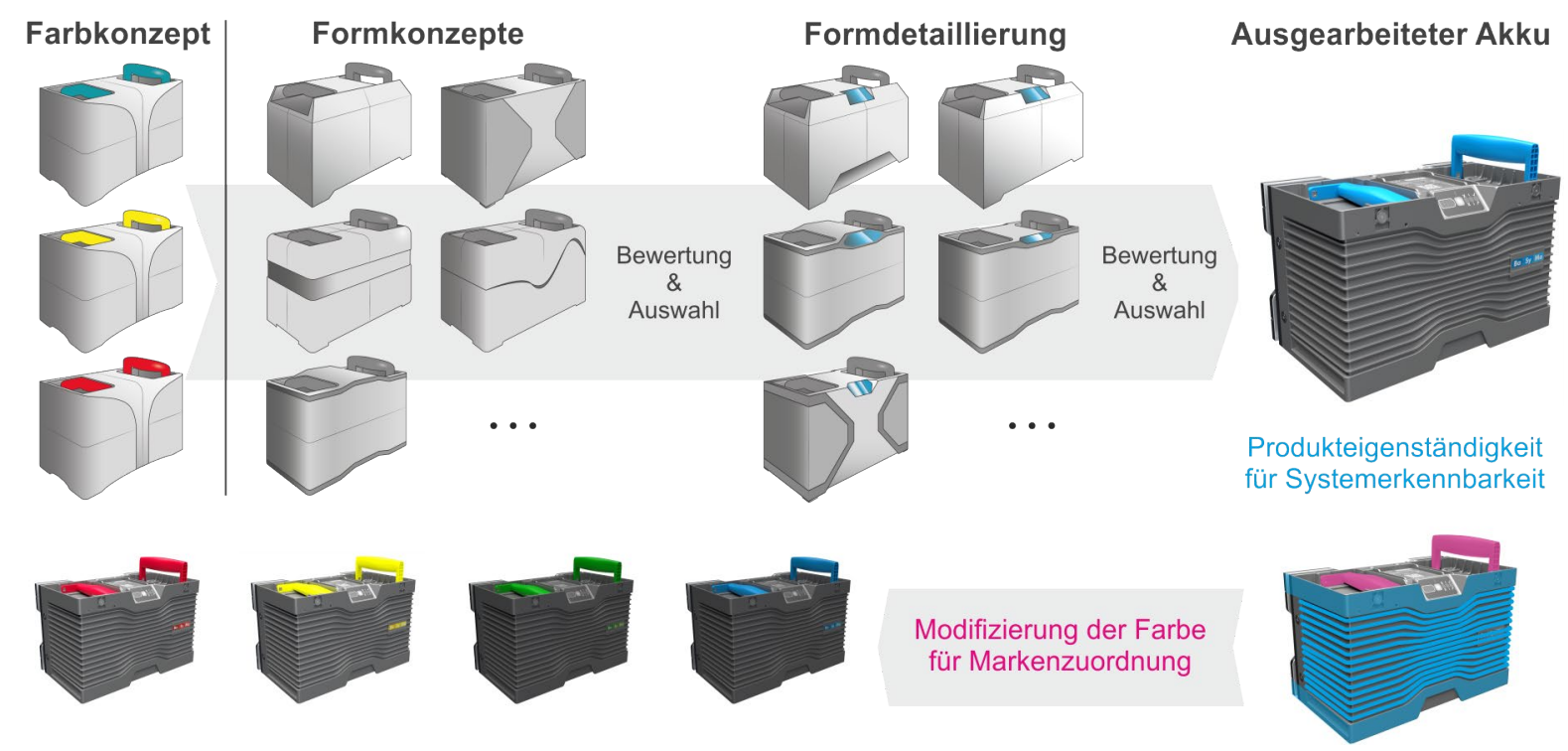

Bild 6: Darstellung des Farbkonzepts, der Formkonzepte und -detaillierungen und des resultierenden markenübergreifenden Produktdesigns am ausgearbeiteten Akku

\section{Zusammenfassung und Ausblick}

Die entwickelte Methode für markenübergreifendes Produktdesign beschreibt das Vorgehen, um Markenzuordnung und Produkteigenständigkeit von Beginn an gezielt zu gestalten. Basis dafür ist der technische Freiheitsgrad für die Gestaltung und eine Wettbewerbsanalyse. Zudem muss entschieden werden, inwiefern das Produktdesign in dem Wettbewerberumfeld platziert werden soll. Die Anwendung dieser Methode erfolgt in der Produktplanungsphase und/oder der Planungsphase in der Produktentwicklung. Daher ergeben sich Auswirkungen auf die momentane Reihenfolge in der Präzisierung der Teilgestalten des Produkts. Relevante Teilgestalten, welche für Markenzuordnung und Produkteigenständigkeit ausgewählt werden, sollten ab Beginn der Konzepterstellung berücksichtigt werden. Zudem liegt der generelle Gestaltungsfokus auf diesen Teilgestalten. Der Lösungsraum der Gestaltung wird dadurch fokussiert. Neben der effizienten Gestaltung ermöglicht die Differenzierung in die Teilgestalten eine transparente Kommunikation der Gestaltungsvorgaben gegenüber Industriedesignern und Entwicklern.

Besonders erforderlich ist diese Methode bei austauschbaren Produkten, welche in Anwendungsmaschinen unterschiedlicher Hersteller (Marken) eingesetzt werden. Hier muss nicht nur eine Wettberberanalyse des zu gestaltenden Produkts durchgeführt werden, sondern es müssen auch die Anwendungsprodukte einbezogen und analysiert werden. Beispielhaft wurde diese Entwicklung in dem BaSyMo-Projekt mit einem wechselfähigen Lithium-lonenAkkumodul durchgeführt. Der Erfolg dieser Gestaltung gegenüber anderen Akkumodulen wird sich im Zuge der Markteinführung zeigen. Generell kann die vorgestellte Methode auf jegliche markenspezifischen Produktgestaltungen angewendet werden, denn damit ist es möglich, den Gestaltungsfokus bereits frühzeitig auch auf die markenspezifischen Gestaltmerkmale zu richten. Einzige Voraussetzung ist, dass bereits ein Wettbewerberumfeld existiert. Zudem sollten Kenntnisse über einzusetzende Fertigungsverfahren und Technologien bekannt sein.

\section{Literaturverzeichnis}

[1] Werwitzke, Cora: Swobbee-Akku-Wechselsystem geht in Regelbetrieb über. 2019 [online]. Verfügbar im Internet: <https://www.electrive.net/2019/12/02/swobbee-akku-wechselsystem-geht-in-regelbetrieb-ueber/> [abgerufen am 27.06.2020].

[2] Seeger, Hartmut: Design technischer Produkte, Produktprogramme und -systeme : Industrial Design Engineering. 2., bearb. und erw. Aufl. Berlin, Heidelberg: Springer Verlag, 2005. 
[3] Holder, Daniel: Gefallensurteil und Blickanalyse zum Fahrzeugdesign zukünftiger Aufbaugestalten anhand einer technischen Prognose. Stuttgart: Universität Stuttgart, Dissertation, 2016.

[4] Holder, Daniel et al.: Integrated Product Gestalt Design Method for the Analysis and Definition of Interface Elements Regarding Exterior and Interior. In: Bagnara, Sebastiano et al. (Hrsg.): Proceedings of the 20th Congress of the International Ergonomics Association (IEA 2018) : Ergonomics in Design, Design for All, Activity Theories for Work Analysis and Design, Affective Design. Cham: Springer Verlag, 2019, S. 888-897.

[5] Richtlinie VDI 2221-1, 2019. Entwicklung technischer Systeme - Modell der Produktentwicklung.

[6] Richtlinie VDI 2221, 1993. Methodik zum Entwickeln und konstruieren technischer Systeme.

[7] Richtlinie VDI/VDE 2424-3, 1988. Industrial Design - Grundlagen, Begriffe, Wirkungsweisen - Der IndustrialDesign-Prozeß.

[8] Watty, Robert; Zimmermann, Christian; Reichert, Gerhard: Zusammenarbeit von Ingenieuren und Designern - die überarbeitete VDI/VDE-Richtlinie 2424. In: Stelzer, Ralph H.; Krzywinski, Jens (Hrsg.): Entwerfen Entwickeln Erleben in Produktentwicklung und Design 2019. Dresden: TUDpress, 2019 (Technisches Design, 12), S. 181-194.

[9] Wohlgemuth, Ulrich: Maschinen Design : Industrieprodukte erfolgreich gestalten. Hamburg: Diplomica Verlag, 2016.

[10] Esch, Franz-Rudolf: Strategie und Technik der Markenführung. 7. vollst. überarb. und erw. Aufl. München: Vahlen, 2012.

[11] Kreuzbauer, Robert; Malter, Alan J.: Embodies Cognition ans New Product Design: Changing Product Form to Influence Brand Categorization. In: Journal of Product Innovation Management 22 (2005), Nr. 2, S. 165176.

[12] Keaveney, Susan M. et al.: The Eyes Have It: How a Car's Face Influences Consumer Categorization and Evaluation of Product Line Extensions. In: Psychology \& Marketing Vol. 29 (2012), Nr. 1, S. 36-51.

[13] Herrmann, Andreas et al.: Alles für die Marke? Produktdesign im Konflikt zwischen einer marken-konformen und einer eigenständigen Produktliniengestaltung. In: Schmalenbachs Zeitschrift für betriebswirtschaftliche Forschung Vol. 59 (2007), Nr. 8, S. 1055-1079.

[14] Andersson, Torbjörn; Warell, Anders: Aesthetic Flexibility in the Management of Visual Product Branding. In: Procedia Manufacturing 3 (2015), S. 2191-2198.

[15] Herrmann, Christoph; Moeller, Günter: Strategisches Industriegüterdesign. In: Herrmann, Christoph et al. (Hrsg.): Strategisches Industriegüterdesign : Innovation und Wachstum durch Gestaltung. Berlin: Springer, 2009, S. 9-37.

[16] Maier, Thomas: Gleichteilanalyse und Ähnlichkeitsermittlung von Produktprogrammen. Stuttgart: Universität Stuttgart, Dissertation, 1993.

[17] Heß, Steffen: Ähnlichkeitsermittlung von Produktsystemen. Stuttgart: Universität Stuttgart, Dissertation, 1999.

[18] Fischer, Matthias Sebastian et al.: Evaluating Similarities in Visual Product Appearance for Brand Affiliation. In: Fukuda, Shuichi (Hrsg.): Advances in Affective and Pleasurable Design : Proceedings of the AHFE 2019 International Conference on Affective and Pleasurable Design. Cham: Springer Verlag, 2020, S. 3-12.

[19] Richtlinie VDI/VDE 2424-2, 1986. Industrial Design - Grundlagen, Begriffe, Wirkungsweisen - Darstellung an Beispielen.

[20] Richtlinie VDI/VDE 2424-1, 1986. Industrial Design - Grundlagen, Begriffe, Wirkungsweisen.

[21] Richtlinie VDI 4506-4, 2013. Strategischer Vertrieb - Markenmanagement mit dem Business-Coach.

[22] Mayer, Silke: Wettbewerbsfaktor Design : Zum Einsatz von Design im Markt für Investitionsgüter. Hamburg: S + W Steuer- und Wirtschaftsverlag, 1996.

[23] Klöcker, Ingo: Produktgestaltung : Aufgabe - Kriterien - Ausführung. Berlin, Heidelberg, New York: Springer Verlag, 1981.

[24] Schmid, Markus; Maier, Thomas: Technisches Interface Design : Anforderungen, Bewertung und Gestaltung. Berlin, Heidelberg: Springer Verlag, 2017.

[25] Macey, Stuart; Wardle, Geoff: H-POINT : The Fundamentals of Car Design \& Packaging. 2. Aufl. Culver City: Design Studio Press, 2014.

[26] Richtlinie VDI 4520-1, 2017. Produktmanagement - Einführung und Grundlagen.

[27] Richtlinie VDI 2221-2, 2019. Entwicklung technischer Systeme - Gestaltung individueller Produktentwicklungsprozesse. 